\title{
DATACIONES ABSOLUTAS PARA EL FOSO 1 DE PERDIGÕES (REGUENGOS DE MONSARAZ, PORTUGAL). REFLEXIONES SOBRE SU CRONOLOGÍA Y TEMPORALIDAD
}

\section{ABSOLUTE DATES FROM DITCH 1 AT PERDIGÕES (REGUENGOS DE MONSARAZ, PORTUGAL). SOME THOUGHTS ABOUT ITS CHRONOLOGY AND TEMPORALITY}

\author{
JOSÉ E. MÁRQUEZ ROMERO* \\ ELENA MATA VIVAR** \\ VÍCTOR JIMÉNEZ JÁIMEZ** \\ JOSÉ SUÁREZ PADILLA**
}

\begin{abstract}
Resumen: En el presente artículo se da a conocer una serie de fechas radiocarbónicas correspondientes, todas ellas, al relleno del foso 1 del yacimiento portugués de Perdigões. La ocasión se aprovecha además para discutir sobre la cronología y la temporalidad de este yacimiento donde, junto a otras instituciones, la Universidad de Málaga viene realizando actuaciones arqueológicas desde el año 2008. La lectura inicial nos advierte de los complejos ritmos de amortización que sufrieron los fosos a lo largo de la prolongada vida del yacimiento y de la necesidad de construir modelos más elaborados que expliquen las conductas de uso y abandono de los recintos de fosos prehistóricos.

Palabras Clave: Cronología, Recintos de fosos, abandono, Neolítico, Edad del Cobre, suroeste Península Ibérica, deposiciones estructuradas, foso.
\end{abstract}

\section{INTRODUCCIÓN}

En la necesaria reorientación, tanto conceptual como metodológica, que precisan los estudios sobre los

* Área de Prehistoria. Facultad de Filosofía y Letras. Universidad de Málaga. Campus de Teatinos s/n. 29071-Malaga. Correo-e: jemarquez@uma.es

** Investigador Proyecto "Arqueología y Patrimonio en los Recintos de Fosos de la Península Ibérica. Perdigões como caso de estudio”
Abstract: This article aims to report the results of a radiocarbon dating programme executed using samples from the filling of Ditch 1 at the Portuguese site of Perdigões. We also discuss the temporality and chronology of the site, where, in collaboration with other scientific institutions, the University of Málaga has been carrying out archaeological fieldwork since 2008. Obtained data apparently suggests a high degree of complexity in the process of ditch filling, in line with the enduring life history of the site, encouraging the introduction of more sophisticated models for explaining use and abandonment behaviours at Prehistoric ditched enclosures.

Keywords: Chronology, Ditched enclosures, abandonment, Neolithic, Copper Age, southwestern Iberian Peninsula, structured depositions, ditch.

denominados yacimientos de fosos de la Prehistoria reciente, ocupa un lugar primordial el análisis de su cronología y de su temporalidad. Así, al natural empeño por datar de forma absoluta cualquier yacimiento (cronología), se suma la tarea no menor de desentrañar la

(Plan Nacional Ministerio de Ciencia e Innovación HAR201021610-C02-01), Correo-e: elemavi@hotmail.com, vjjaimez@yahoo. es, psuarezarqueo@gmail.com 


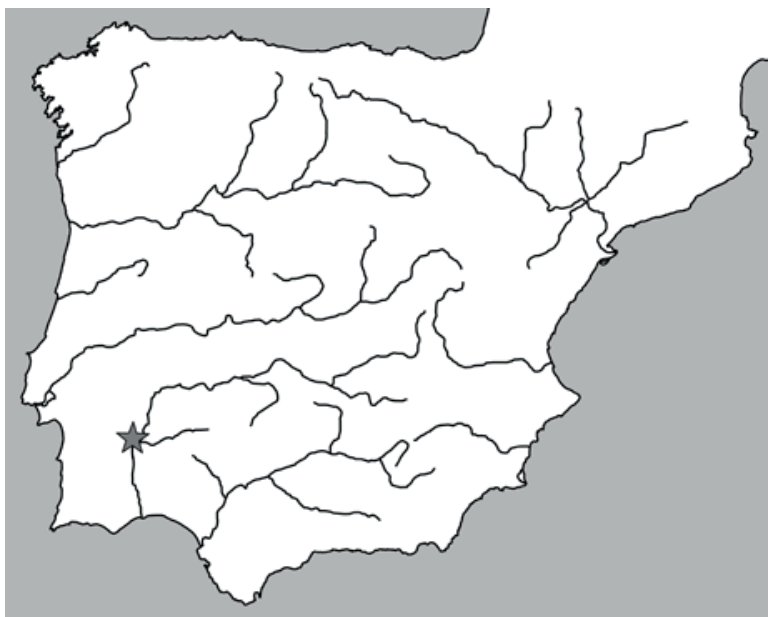

Figura 1. Ubicación del yacimiento de Perdigões (Reguengos de Monsaraz, Portugal).

secuencia de eventos o acontecimientos que está en el centro de la biografía o historia ocupacional de estos complejos yacimientos (temporalidad); en suma, las relaciones de anterioridad, posterioridad y contemporaneidad entre los elementos que los forman.

Los yacimientos "negativos" (recintos de fosos y campos de hoyos) presentan dinámicas específicas en la formación de su registro arqueológico que los distinguen de cualquier otro lugar prehistórico. Hablamos de reiteradas prácticas de fundación, uso, condenación y/o abandono de tales sitios, que quedan concretadas en la excavación y posterior relleno de multitud de estructuras negativas. Este hecho lo hemos explicado (Márquez y Jiménez 2010: cap. 11) como consecuencia de los patrones de asentamiento y abandono de lugares que, definidos por una marcada estacionalidad, son propios de una gran parte de poblaciones de la Prehistoria reciente del occidente europeo (p.e. Cunliffe 1992, Brück 1999, Pollard 1999, 2001, Chapman 2000, Garrow et al. 2005, Anderson-Whymark y Thomas 2012). La presencia recurrente de deposiciones estructuradas en el interior de fosas o en las colmataciones de los grandes fosos, entre otros indicadores, nos sugiere que la intencionalidad antrópica está en la génesis de este fenómeno (Márquez y Jiménez 2010: caps. 9-10).

En este escenario, nos interesan los ritmos de colmatación, la contemporaneidad de unas estructuras y otras, los solapamientos y recortes (recuttings), etc. pues nos informan de la temporalidad del lugar y de la manera como el tiempo-espacio, vivido como presente, se puede enlazar con lo ya acontecido o lo que inminentemente va a acontecer en el mismo lugar. Sólo desde la evaluación de este tiempo corto o événementielle (Braudel 1986: 66), esto es, de la secuenciación de acontecimientos que quedan arqueológicamente fosilizados en el terreno, podremos alcanzar la comprensión real de estos yacimientos y la intencionalidad humana que los generó (Márquez y Jiménez e.p.).

\section{EL COMPLEXO ARQUEOLÓGICO DOS PERDIGÕES}

El Complexo Arqueológico dos Perdigões es un yacimiento que se localiza en el Concelho de Reguengos de Monsaraz, a unos $2 \mathrm{~km}$ al NW del núcleo urbano, en el distrito de Évora, en el Alentejo portugués (fig. 1). Comprende, básicamente, una serie concéntrica de recintos prehistóricos de fosos y empalizadas, a la que se puede asociar un espacio de necrópolis ubicado entre los fosos 1 y 2 y los restos de un posible crómlech localizado en la periferia oriental de los citados recintos (fig. 2). El área total ocupada por el yacimiento alcanza 16 ha. La investigación en el sitio se remonta a los años 90 del pasado siglo (Gomes 1994) y desde entonces se han realizado numerosas actuaciones arqueológicas (Lago et al. 1998, Valera 2008a-b, 2009, Valera et al. 2008). En el año 2008, el Área de Prehistoria de la Universidad de Málaga se sumó a los trabajos, desarrollando un proyecto propio que se integra, como un subprograma, en el marco general coordinado por el NIA de Era Arqueología (Márquez et al. 2008, 2011c). Los objetivos de este subprograma han sido adelantados en su momento (Márquez et al. 2008). No obstante, cabe aquí recordar que uno de los objetivos principales de nuestras actuaciones es:

Objetivo 3.2. Encontrar o descartar relaciones de temporalidad tanto a nivel interno, dentro de cada una de las estructuras, como entre ellas (especialmente los fosos exteriores), relacionando estas cronologías a su vez con las procedentes del área de necrópolis. (Márquez et al. 2008: 29).

Consecuentemente con lo apuntado, desde esos momentos nuestras actuaciones han estado orientadas, preferentemente, a establecer la cronología comparada de los dos fosos paralelos y exteriores del yacimiento ( 1 y 2) con el fin de confirmar o no su posible contemporaneidad (objetivo 3.2.1); también buscamos aclarar la temporalidad interna dentro de cada foso o estructura (objetivo 3.2.2), así como relacionar las cronologías logradas en las zanjas exteriores, tanto con aquellas que puedan obtenerse en la zona de necrópolis megalítica 


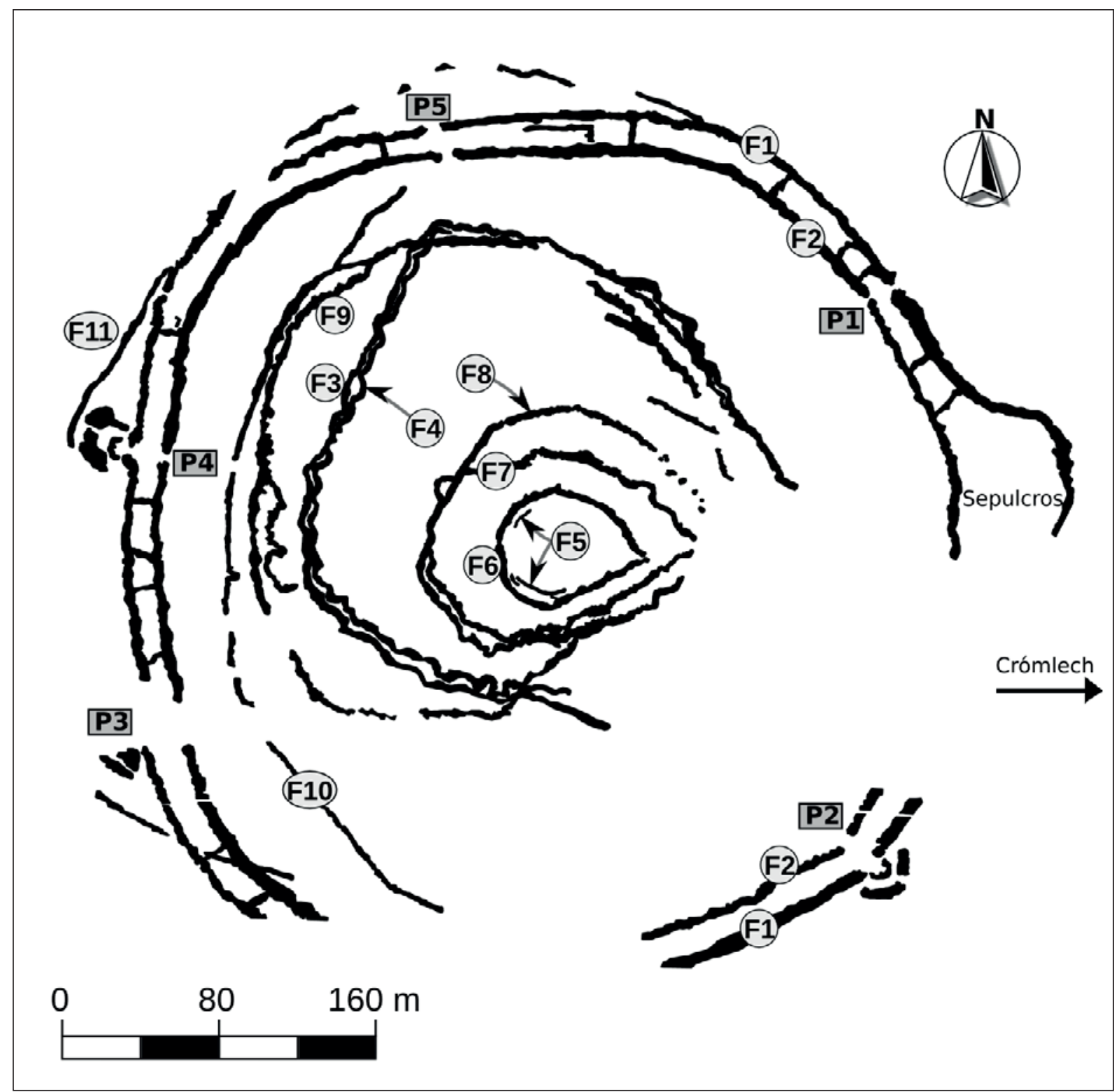

Figura 2. Yacimiento de Perdigões con indicación de fosos y puertas. Realizado a partir de prospecciones geomagnéticas.

(objetivo 3.2.3), como en aquellas áreas interiores del yacimiento en las que están trabajando otras instituciones científicas (objetivo 3.2.4).

En este empeño, presentamos ahora, y de forma preliminar, las primeras dataciones absolutas obtenidas por nuestro equipo en el yacimiento de Perdigões. Proceden todas ellas del relleno del foso 1, en un tramo próximo a la puerta NE (puerta 1) del recinto exterior (sector L), zona específica donde se han concentrado los trabajos de la Universidad de Málaga durante el trienio 2009-2011.

\section{EL FOSO 1 DEL COMPLEXO DOS PERDIGÕES}

En 1997, Era-Arqueología comenzó la excavación de un tramo del denominado foso 1 de este importante yacimiento alentejano, pero dicha actuación no pudo ser finalizada (Lago et al. 1998). Se ubicaba el tramo de foso excavado muy próximo a la puerta 1 , que ya en aquellos momentos se conocía por la fotografía aérea tomada del yacimiento. Las prospecciones geofísicas llevadas a cabo en 2009 (Márquez et al. 2011d) 
confirmaron la existencia de dicha puerta y animaron al equipo de la UMA a retomar los trabajos en la zona. Pero, en esta coyuntura, no parecía legítimo abrir un corte nuevo, con lo que de gastos e impacto en el yacimiento supondría, apenas a unos metros de distancia de un sondeo no finalizado con anterioridad. Esto hizo que durante dos campañas, concretamente en 2009 y 2010, se reabriera y ampliara el corte L1 de 1997 y se retomara, hasta su fondo, la excavación de dicho foso 1 . La decisión creemos que, en líneas generales, fue acertada. La optimización de recursos y el respeto por la conservación del yacimiento que la justificaba no menguaron los objetivos alcanzados. No obstante, como contrapartida, tal decisión nos obligó a realizar una serie de ajustes metodológicos concretos, puesto que los objetivos de partida de las campañas de 1997 (Lago et al. 1998) y de 2009-2010 (Márquez et al. 2008, Márquez et al. 2011b) eran bien distintos. Por otro lado, el sondeo de 1997, al no disponer en aquellos momentos de la planimetría precisa aportada por las prospecciones geofísicas, se realizó con orientación estricta norte-sur, lo que provocó que el corte cruzara, sin pretenderlo, el foso 1 de forma oblicua y no perpendicular como hubiera sido deseable, y tal y como se pudo replantear, con posterioridad, en las campañas 2009 y 2010. Como consecuencia, en la actualidad, no disponemos de un perfil completo del relleno del foso 1 . A efectos prácticos, y dada la naturaleza de estos rellenos, resulta un problema menor, pero dificulta una representación gráfica al uso del mismo. Las secciones de ambos tramos están publicadas por separado y remitimos al lector a dichos trabajos (Lago et al. 1998, Márquez et al. 2011b) mientras que en el presente utilizaremos la matriz de Harris como soporte de la exposición.

Ciñéndonos a las dos campañas de trabajo de la Universidad de Málaga, la estratigrafía interior resultante nos confirmó el carácter eminentemente antrópico de los procesos formativos de la mayoría de depósitos arqueológicos que configuraban el relleno del foso. Por otra parte, se pudo discriminar la existencia de dos fases o dinámicas estratigráficas distintas en dicho proceso de rellenado, cada una con sus propios ritmos (Márquez et al. 2011b: 171-172). En resumen podrían caracterizarse de la siguiente manera.

a) Fase I. La más profunda, se inicia con un nivel estéril de poca profundidad (UE 140) sobre el que se superponen lo que podríamos denominar "depósitos iniciales" o de "fundación", en los que se combinan, de forma significativa, restos óseos, cantos y material arqueológico (UU.EE 139 a/b y 134). A ellos sucede un depósito homogéneo, arenoso y arqueológicamente estéril (UE 129=136), formado posiblemente durante un episodio hídrico en el yacimiento, sobre el que se excavaron una serie de pequeñas fosas, en algunos casos superpuestas entre sí (UU.EE. 131, 137, $138,128)$. Cada una de estas fosas, cubiertas a su vez por la UE 122, contenía deposiciones reconocibles. Sobre estos niveles se vuelven a constatar un nuevo episodio presumiblemente natural (UE 123), de naturaleza semejante a la referida UE $129=136$ y que cierra "a techo" esta fase inicial, que apenas si acumula $70 \mathrm{~cm}$ de sedimentos.

Probablemente, se debió formar como consecuencia de sucesivas deposiciones deliberadas, llevadas a cabo desde dentro del propio foso, en un momento en el cual éste era transitado.

b) Fase II. Sobre las UU.EE 122 y 123, se identifica un paquete de estratos bien distinto a los subyacentes (fase I), con cerca de $2 \mathrm{~m}$ de potencia, y alto contenido en restos faunísticos, cerámicos y grandes bloques de piedras. Están documentados tanto en las excavaciones de 2009-2010 (UU.EE. 118 y 116) como en la mayoría de las unidades estratigráficas excavadas en 1997 por Era-Arqueologia (UU.EE. 54, 50, 51, 31, 37, 11, 12). Configura una serie de estratos de gran potencia y, en muchos casos, con una marcada inclinación, que parece responder a un relleno rápido efectuado por agentes humanos, sobre todo, desde fuera del foso. Algunos indicios (aún débiles) apuntan a que, sobre este potente paquete identificado como fase II, y una vez que quedó totalmente colmatado el foso 1 , se realizó, a modo de recutting, una fosa alargada excavada directamente sobre los sedimentos de esta fase II.

\section{DATACIONES ABSOLUTAS DEL FOSO 1 DEL COMPLEXO DOS PERDIGÕES}

Hasta el momento presente, las dataciones absolutas procedentes del yacimiento de Perdigões se concentraban en sectores interiores. Concretamente 8 fechas habían sido obtenidas en el sector I (en los fosos 3 y 4 , y en las fosas 7 y 11) y una más en el sector Q (en la fosa 4) (Valera y Silva 2011: 10). Tras la excavación definitiva del foso 1 , en el sector más externo del yacimiento, podemos incorporar una nueva batería de nueve dataciones absolutas (tab. 1). Inicialmente fueron enviadas al laboratorio Beta hasta 11 muestras, todas pertenecientes a restos óseos. Dos de ellas fueron descartadas sin llegar a datarse, en un caso al no 
Tabla 1. Resultado de las dataciones de radiocarbono realizadas en el foso 1 mediante AMS. Calibradas a partir de INTCAL09 (Oeschger et al. 1975, Stuiver y Braziunas 1993, Heaton et al. 2009).

\begin{tabular}{|c|c|c|c|c|c|c|c|}
\hline Ref. Lab. & Estruct. & UE & Naturaleza muestra & Data BP & Data BC & Cal BC $1 \sigma$ & Cal BC $2 \sigma$ \\
\hline Beta-315717 & Foso 1 & 12 & $\begin{array}{c}\text { Astrágalo } \\
\text { Cervus elaphus }\end{array}$ & $3980 \pm 30$ & $2030 \pm 30$ & $2560-2470$ & $2570-2460$ \\
\hline Beta-315716 & Foso 1 & 11 & $\begin{array}{c}\text { Húmero } \\
\text { Sus sp }\end{array}$ & $3770 \pm 30$ & $1820 \pm 30$ & $2270-2140$ & $2290-2060$ \\
\hline Beta-315718 & Foso 1 & 31 & $\begin{array}{c}\text { Diente } \\
\text { Sus sp }\end{array}$ & $4060 \pm 30$ & $2110 \pm 30$ & $2620-2500$ & $2830-2490$ \\
\hline Beta-315720 & Foso 1 & 116 & $\begin{array}{c}\text { Diente } \\
\text { Ovis/Capra }\end{array}$ & $3860 \pm 30$ & $1910 \pm 30$ & $2430-2290$ & $2460-2200$ \\
\hline Beta-315719 & Foso 1 & 118 & $\begin{array}{c}\text { Diente } \\
\text { Sus sp }\end{array}$ & $3780 \pm 30$ & $1830 \pm 30$ & $2280-2140$ & $2290-2140$ \\
\hline Beta-315721 & Foso 1 & 122 & $\begin{array}{c}\text { Hueso especie } \\
\text { indeterminada }\end{array}$ & $3840 \pm 30$ & $1890 \pm 30$ & $2340-2210$ & $2460-2200$ \\
\hline Beta-315722 & Foso 1 & 133 & $\begin{array}{c}\text { Diente } \\
\text { Sus sp }\end{array}$ & $3890 \pm 30$ & $1940 \pm 30$ & $2460-2300$ & $2470-2290$ \\
\hline Beta-315723 & Foso 1 & 134 & $\begin{array}{c}\text { Hueso especie } \\
\text { indeterminada }\end{array}$ & $3820 \pm 30$ & $1870 \pm 30$ & $2290-2200$ & $2400-2150$ \\
\hline Beta-315725 & Foso 1 & 139 & $\begin{array}{l}\text { Hueso especie } \\
\text { indeterminada }\end{array}$ & $3890 \pm 30$ & $1940 \pm 30$ & $2460-2300$ & $2470-2290$ \\
\hline
\end{tabular}

contener suficiente colágeno y en otro al presentar indicios muy evidentes de contaminación.

Detallaremos a continuación la naturaleza de cada una de las muestras y el contexto preciso en el que fueron extraídas (para una información completa de la secuencia, véase Márquez et al. 2011b). Se describen desde las muestras obtenidas en las UEs más superficiales a las más profundas (tab. 2).

a) Beta-315717: $3980 \pm 30$ BP (2560-2470 Cal BC, $1 \sigma)$. Muestra obtenida a partir de un fragmento de astrágalo de Cervus elaphus. Recogida durante la campaña de 1997, en la UE 12 del Sector 5 por ERA-Arqueologia. Analizada por AMS. La UE 12 es un nivel con abundante material arqueológico, sobre todo cerámico, cuya génesis, según se pensó en 1997, se debía a la lenta sedimentación natural de tierras y artefactos (Lago et al. 1998: 71).

b) Beta-315716: $3770 \pm 30 \mathrm{BP}(2270-2140 \mathrm{BC}, 1 \sigma)$. Muestra obtenida a partir un fragmento de húmero de Sus sp. Recogida durante la campaña de 1997, en la UE 11 del Sector 5 por ERA-Arqueologia. Analizada por AMS. Este estrato se caracteriza por ser un conjunto de piedras de mediano y gran tamaño, interpretado en su momento como parte del derrumbe de una estructura de piedra adyacente al foso (Lago et al. 1998: 71-72), hoy descartada esa posibilidad al no haberse constatado restos de estructuras emergentes en las inmediaciones del foso. Aparecieron dos fragmentos de cerámica con decoración campaniforme incisa (Albergaria 1998: 114115). Se asentaba sobre la UE 31 y algunos puntos de la UE 37, apoyándose sobre la pared norte del foso. Estaba cubierto por la UE 12.

c) Beta-315718: 4060 $\pm 30 \mathrm{BP}(2620-2500 \mathrm{BC}, 1 \sigma)$. Muestra obtenida a partir de dos fragmentos de dientes de Sus sp. Recogida durante la campaña de 1997, en la UE 31 del Sector 5 excavado por ERAArqueologia. Analizada por AMS. Este estrato fue interpretado en 1997 como un sedimento de origen natural que integraba abundantes restos cerámicos y faunísticos (Lago et al. 1998: 71). Aparecieron tres fragmentos de cerámica con decoración campaniforme incisa (Albergaria 1998: 114-115). Cubría a la UE 74 y estaba cubierto por la UE 11.

d) Beta-315720: $3860 \pm 30 \mathrm{BP}(2430-2290 \mathrm{BC}, 1 \sigma)$. Muestra de hueso obtenida a partir de un diente 
completo de Ovis/Capra. Recogida durante la campaña de 2009, en el subsector A del Sondeo L-1, UE 116. Analizada por AMS. El estrato UE 116 se corresponde con el relleno de una posible fosa alargada realizada sobre rellenos preexistentes del foso (recutting), con tendencia en "U" y sensiblemente paralela al eje principal del foso. Llega incluso a cortar parte del nivel UE 123. Su potencia máxima documentada fue de $60 \mathrm{~cm}$, y su anchura alcanzó los $87 \mathrm{~cm}$. Si algo caracterizó su relleno, fue la aparición de abundantes bloques líticos de tamaño grande y mediano (granitos, dioritas y esquistos), a los que se une una significativa presencia de restos faunísticos (incluidas algunas defensas de bóvidos) y de cerámica. Este mismo depósito fue documentado en la campaña de 1997, aunque entonces se denominó UE 74.

e) Beta-315719: 3780 $\pm 30 \mathrm{BP}(2280-2140 \mathrm{BC}, 1 \sigma)$. Muestra obtenida a partir de un diente casi completo de Sus. sp., recogida durante la campaña de 2009, en el subsector A del Sondeo L-1, UE 118. Analizada por AMS, la UE 118 resultó ser un nivel formado sobre la UE 123, generalizado en toda la superficie excavada, apoyándose sobre las paredes del foso. Este estrato es a su vez cortado por la base de la fosa antes citada UE 116. Presenta matriz areno-arcillosa, algo heterogénea y semi-compacta, con algunos nódulos de matriz geológica descompuesta. Contiene abundantes restos faunísticos, piedras de tamaño medio-pequeño, restos cerámicos, y presencia puntual de restos de adobe, muy degradados. Este estrato excavado por la UMA en la campaña 2009 se correspondería con la UE 28 excavada por ERA-Arqueologia durante su campaña de 1997, nivel en el que fue documentado un fragmento de cerámica con decoración puntillada e incisa campaniforme (Albergaria 1998: 114-115).

f) Beta-315721: $3840 \pm 30 \mathrm{BP}(2340-2210 \mathrm{BC}, 1 \sigma)$. Muestra de hueso obtenida a partir de un fragmento de costilla de un animal de medio porte (indeterminado). Recogida durante la campaña de 2010, en el subsector A del Sondeo L-1, UE 122. Analizada por AMS. El nivel UE 122 es aquel que definitivamente amortiza el estrato UE $129=136$, una vez realizada las fosas que lo cortan. Este nivel de $11 \mathrm{~cm}$ de potencia se generaliza en toda la planta excavada, apoyando sobre las paredes del foso. Además, aparece cubierto parcialmente por un estrato de naturaleza semejante a UE 129=136, la UE 123. Entre las inclusiones que contiene dominan los fragmentos de vajilla cerámica, junto a restos faunísticos, algún canto rodado y presencia testimonial de restos de adobe, muy degradados.

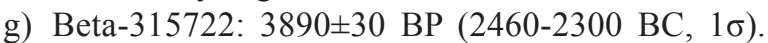
Muestra de hueso obtenida a partir de un fragmento de diente de Sus. sp. Recogida durante la campaña de 2010, en el subsector A del Sondeo L-1, UE 133. Analizada por técnica de AMS. La UE 133 se corresponde con el relleno de la primera de las fosas que cortaban al estrato estéril UE 129=136. De este nivel se conservaron $7 \mathrm{~cm}$ de grosor, aunque en origen debió de presentar una mayor potencia. Este estrato apareció cortado en su sector meridional por otra fosa, la UE 135. Además, su techo es también cortado por la fosa UE 131. En su relleno fueron recuperados fragmentos de vajilla cerámica.

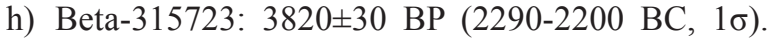
Muestra de hueso obtenida a partir de un fragmento de hueso de animal de medio porte (indeterminado). Recogida durante la campaña de 2010 , en el subsector A del Sondeo L-1, UE 134. Analizada por técnica de AMS. El nivel UE 134, de $14 \mathrm{~cm}$ de potencia, se localiza sobre la UE 139 (subnivel 139b). A su vez, está cubierto por el estrato UE $129=136$, de naturaleza bien distinta, al ser consecuencia de la aparente descomposición de los gabrodioritos que forman las propias paredes del foso. Presenta restos cerámicos y faunísticos, así como un fragmento de un pequeño objeto de cobre no identificable. Se caracteriza por contener nódulos anaranjados de arcilla, resultantes de la descomposición de adobes, así como por tener un mayor número de carbones en comparación con otros estratos de la secuencia.

i) Beta-315725: $3890 \pm 30 \mathrm{BP}(2460-2300 \mathrm{BC}, 1 \sigma)$. Muestra de hueso obtenida a partir de un fragmento de costilla de un animal de gran porte (indeterminado). Recogida durante la campaña de 2010, en el subsector A del Sondeo L-1, UE 139. Analizada por técnica de AMS. Corresponde al primero de los estratos de colmatación del Foso 1, sobre la estéril UE 140. Con una potencia de $15 \mathrm{~cm}$, presentaba inclusiones en su matriz de pequeños restos de adobe, partículas de carbón y algunas rocas de diversa naturaleza como esquistos que en algún caso presentaban signos de termoalteración. El estrato presentaba restos cerámicos y faunísticos, con una peculiar disposición en tongadas dentro de la misma matriz, diferenciándose, por tanto, dos subniveles: 139a (inferior) y $139 \mathrm{~b}$ (superior). 
Tabla 2. Dataciones absolutas obtenidas en el foso 1 de Perdigões con indicación de la UEs de procedencia de cada una de ellas.

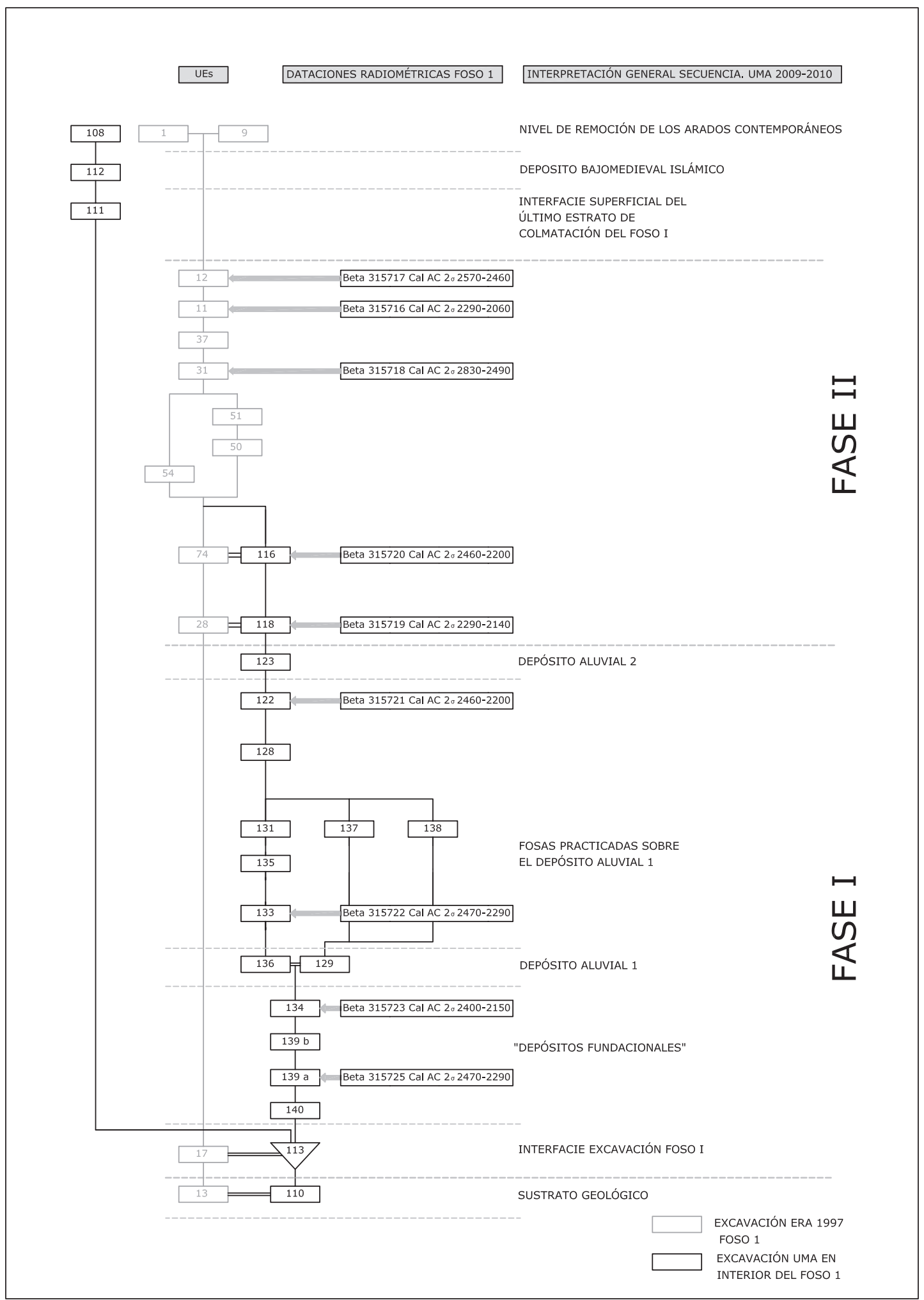

ISSN: $1133-4525$ ISSN-e: $2255-3924$ 


\section{CONSIDERACIONES FINALES}

Resulta procedente realizar, ahora, una lectura preliminar de los datos radiométricos obtenidos en el foso 1 de Perdigões. Para ello nos serviremos de las propias dataciones absolutas (tab. 1), de su correspondencia estratigráfica (tab. 2) y de la comparativa con los datos obtenidos previamente en los fosos 3 y 4 del mismo yacimiento (fig. 3) (Valera y Silva 2010). Por el contrario hemos renunciado, en este momento, a abordar una comparativa más amplia en la que se relacionaran los datos radiométricos aquí obtenidos con los procedentes de otros yacimientos de fosos del mediodía peninsular. Pensamos que resulta poco operativo y puede llevar a conclusiones precipitadas comparar fechas aisladas recogidas en yacimientos donde no se ha podido resolver la temporalidad de las estructuras que lo configuran. Por el contrario reclamamos que la tarea previa para avalar estas comparaciones a gran escala pasa ineludiblemente por la realización de amplias series de dataciones y por la reconstrucción biográfica de cada yacimiento, tal y como se está abordando en otras áreas europeas (Whittle et al. 2011) y como ya se ha iniciado en el propio yacimiento de Perdigões (p.e. Valera y Silva 2010, Márquez et al. 2011a, Valera et al. e.p.). El análisis de lo que ocurre en el relleno del foso 1, que expondremos a continuación, nos convencerá de la complejidad de los procesos de formación del registro arqueológico en estos yacimientos y de la cautela que debemos tener al valorar, simplistamente, una fecha aislada dentro del complicado proceso que va desde la construcción de la estructura hasta su relleno definitivo.

En cualquier caso, el tema del origen y la tradición de los recintos de fosos y su proyección cronológica en el proceso histórico del suroeste de la Península Ibérica (VI-III milenios a.C.) ha sido abordada en profundidad y con el detenimiento que merece en otro momento (Márquez y Jiménez 2010: cap.11) por lo que remitimos al lector interesado a la consulta de dicho trabajo.

Tras estas aclaraciones previas, podemos adelantar, aunque sea de forma provisional, que en el relleno del foso 1 se observa:

a) Una fase I, la más profunda, con $70 \mathrm{~cm}$ de potencia total, comprendida entre las UU.EE. 140 (a muro) y 123 (a techo), ambas estériles. De ella poseemos hasta 4 fechas, que sitúan el relleno y, quizás, la propia construcción del foso, en el tercer cuarto del III milenio a.C. El depósito aluvial 1 (UE 136=129) no parece ser significativo ni a nivel estratigráfico ni cronométrico pues no supone un cambio entre las unidades subyacentes y las que se le superponen.
Por otra parte, la nítida estratigrafía con la identificación de sucesivas deposiciones estructuradas y la coherencia de las cuatro dataciones nos habla de una fase "de uso" en la que personas transitaron por el interior del foso, rellenándolo desde el interior de forma normalizada (Márquez et al. 2011b: 172).

b) Una fase II, de gran potencia, más de $2 \mathrm{~m}$ de relleno, que se superpone al depósito aluvial 2 (UE 123). De ella hemos obtenido las cinco dataciones restantes, que resultan, sin lugar a dudas, de más compleja interpretación. Por una parte, contamos con dos dataciones, prácticamente idénticas, Beta 315719 en la UE 118 (a muro de esta fase II) y Beta 315716 en la UE 11 (prácticamente a techo de la misma fase II), que parecen fechar este momento de colmatación del foso a comienzos del último cuarto del III milenio a.C. Ello supondría, en cualquier caso, un momento algo más tardío que la construcción del foso $1 \mathrm{y}$ el proceso de relleno durante la fase I o de "uso". Como ya hemos comentado con anterioridad, esta fase II parece ser el resultado de una colmatación rápida realizada desde el exterior del foso en la que se incluían grandes bloques de piedra y abundante fauna, provocando una dinámica muy diferente a la de los niveles más profundos. Su fisonomía y ritmo de colmatación nos invitan, con todas las cautelas, a identificarla como una posible "fase de abandono" (Márquez et al. 2011b: 172).

Pero las tres fechas restantes distorsionan la coherencia cronológica de esta fase II, pues son más antiguas, no sólo que este momento final del relleno, sino incluso que las recuperadas en la fase I, que desde el punto de vista estratigráfico se formaron antes. Esta contingencia, en el momento actual, podría explicarse de distinta manera:

1) La introducción de forma incontrolada y residual de restos de fauna, mucho más antiguos, procedentes de otros contextos del yacimiento, durante la definitiva colmatación (fase II) del foso 1 , o durante un posible recutting posterior (Márquez et al. 2011b: 169-170).

2) La introducción ex profeso de restos óseos animales que previamente fueron extraídos del relleno de estructuras más antiguas del yacimiento. Esta conducta ya ha sido apuntada para explicar un comportamiento similar en el foso 4 (Valera y Silva 2011: 9) y nos estaría hablando de procesos muy complejos de relleno de los fosos en los que la secuencia de acontecimientos y la temporalidad es mucho más complicada de la inicialmente considerada. 


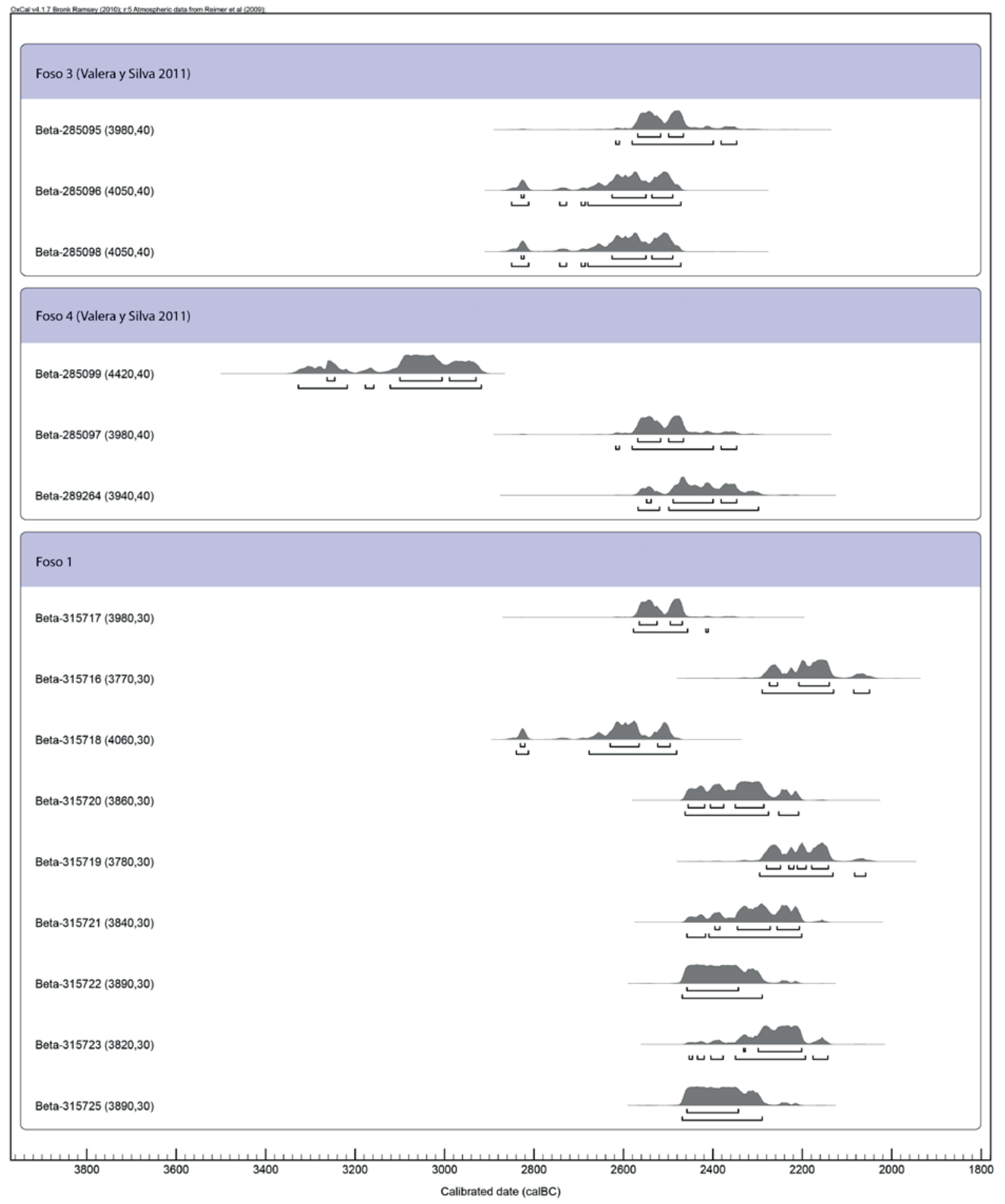

Figura 3. Comparativa entre las dataciones del foso 1 y las procedentes de los fosos 3 y 4 (Valera y Silva 2011). 
c) Si comparamos las dataciones del foso 1 con las previamente obtenidas en los fosos 3 y 4 (Valera y Silva 2011), observaremos (fig. 3) que no son contemporáneas, al menos en lo que se refiere a su colmatación, confirmando que el dibujo de la planta del sitio que nos han aportado tanto las fotografías aéreas como las prospecciones geomagnéticas es acumulativo. Podemos pensar, por los indicios que tenemos en este momento, que cuando unos fosos estaban abiertos otros hacía tiempo que se habían cerrado (Márquez et al 2011 a: 577).

d) Los fosos 1, 3 y 4 se construyen, aparentemente, de forma sucesiva y escalonada a partir del segundo cuarto del III milenio a.C. Esto apunta a una cronología calcolítica muy avanzada para las fases finales del yacimiento y lo coloca como uno de los yacimientos con recintos de fosos más tardíos de la Península Ibérica y, con ello, de toda Europa occidental.

e) Por último, cabe apuntar que la existencia de dos fases en el relleno no es exclusiva del foso 1; se documentó algo semejante respecto del foso 3 (Valera y Silva 2011: 11). Ello nos sugiere una dinámica de colmatación compleja en la que, tras una fase "de uso", las deposiciones desde el interior de los fosos cesarían, sin que ello, no obstante, supusiera su colmatación definitiva. En este escenario, y tras un periodo de tiempo de difícil concreción pero que pudo quedar testimoniado por dos momentos de erosión hídrica (UE 58 en el foso 3 y UE 123 en el foso 1), se realizaría la colmatación definitiva de las estructuras, quizá coincidiendo con la construcción de otro foso o fosos vecinos. Es decir, la temporalidad de la construcción de cada foso y su pertinente relleno pudo estar relacionada con los de otras estructuras contemporáneas o más antiguas (Valera y Silva 2011: 12).

Sin lugar a dudas, el panorama que las primeras dataciones de Perdigões nos están mostrando es tremendamente complejo y matizan, al menos en este yacimiento portugués, los modelos teóricos iniciales que propusimos para explicar el relleno de los fosos en estos yacimientos (Márquez 2003: 276) y que con el tiempo se nos están mostrando demasiado simples.

\section{AGRADECIMIENTOS}

Todos los análisis radiocarbónicos han sido sufragados con los recursos procedentes del Proyecto del Plan Nacional del Ministerio de Ciencia e Innovación titulado "Arqueología y Patrimonio en los Recintos de Fosos de la Península Ibérica. Perdigões como caso de estudio" (HAR2010-21610-C02-01). Por otra parte, la colaboración de Era Arqueología y su disponibilidad para permitirnos el acceso a los materiales y a la documentación de la intervención de 1997 ha sido imprescindible para la realización de este trabajo. Agradecemos especialmente la predisposición y ayuda de António Valera, Lucy Evangelista y Miguel Lago.

\section{BIBLIOGRAFÍA}

Albergaria, J. (1998): “Recipientes cerámicos campaniformes recolhidos no Povoado dos Perdigões", en M. Lago, A.C. Valera, C. Duarte, J. Albergaria, F. Almeida, A.F. Carvalho, y S. Reis (eds.), Povoado Pré-Histórico dos Perdigões (Reguengos de Mosaraz). Era 1, Relatório final dos trabalhos de salvamento arqueológico, pp. 106-128. Lisboa, ERA-Arqueologia.

Anderson-Whymark, H. y Thomas, J. (2012): Regional perspectives on Neolithic pit deposition: beyond the mundane. Neolithic studies group seminar papers 12. Oxford y Oakville, Oxbow Books.

Braudel, F. (1986): La Historia y las Ciencias Sociales. Madrid, Alianza Editorial.

Brück, J. (1999): "What's in a settlement? Domestic practice and residential mobility in Early Bronze Age southern England", en J. Brück y M. Goodman (eds.), Making places in the prehistoric world: themes in settlement archaeology, pp. 52-75. Londres, UCL Press.

Cunliffe, B. (1992): "Pits, preconceptions and propitiation in the British Iron Age". Oxford Journal of Archaeology II(I): 69-83.

Chapman, J. (2000): "Pit-digging and Structured Deposition in the Neolithic and Copper Age". Proceedings of the Prehistoric Society 66: 61-87.

Garrow, D.; Beadsmoore, E. y Knight, M. (2005): "Pit clusters and the temporality of occupation: an Earlier Neolithic site at Kilverstone, Thetford, Norfolk", Proceedings of the Prehistoric Society 71: 139-157.

Gomes, M.V. (1994): "Menires e cromeleques no complexo cultural megalítico português - trabalhos recentes e estado da questão", en Actas del seminario "O Megalitismo no Centro de Portugal: novos dados, problemática e relações com outras áreas peninsulares" (Mangualde 1992), pp. 317-342. Viseu, Centro de estudos Pré-Históricos da Beira Alta. 
Heaton, T.J.; Blackwell P.G. y Buck C. E. (2009): “A Bayesian approach to the estimation of radiocarbon calibration curves: the IntCal09 methodology". Radiocarbon 51(4): 1151-1164.

Lago, M.; Duarte, C.; Valera, A., Albergaria, J.; Almeida, F. E. y Carvalho, A. (1998): "Povoado dos Perdigões (Reguengos de Monsaraz): dados preliminares dos trabalhos arqueológicos realizados em 1997". Revista Portuguesa de Arqueologia 1(1): 45-152.

Márquez, J.E. (2003): “Recintos prehistóricos atrincherados (RPA) en Andalucía (España): una propuesta interpretativa", en S. Jorge Oliveira (coord.) Recintos murados da Pré-historia Recente, CoimbraPorto 2002, pp. 269-284.

Márquez, J.E. y Jiménez, V. (2010): Recintos de Fosos. Genealogía y significado de una tradición en la Prehistoria del suroeste de la Península Ibérica (IV-III milenios a.C.). Málaga, Servicios de publicaciones de la Universidad de Málaga.

Márquez, J.E. y Jiménez, V. (en prensa): "Space and time in the architecture of prehistoric enclosures. The Iberian Peninsula as a case study", en S. Souvatzi y A. Hadji (eds.), Space and Time in Mediterraean prehistory, Routledge, Londres-Nueva York.

Márquez, J.E., Jiménez, V. y Mata, E. (2008): “Excavaciones en el yacimiento de Perdigões (Reguengos de Monsaraz, 2008-2010)". Universidad de Málaga (España)". Apontamentos de Arqueologia e Património 2: 41-48.

Márquez, J.E.; Jiménez, V. y Suárez, J. (2011a). “Deconstruyendo Perdigões. Sobre la temporalidad en los yacimientos de fosos del sur de la Península Ibérica", en Memorial Luis Siret. I Congreso de Prehistoria de Andalucía. La tutela del patrimonio histórico, pp. 575-578. Sevilla, Junta de Andalucía.

Márquez, J.E.; Suárez, J.; Jiménez, V. y Mata, E. (2011b): “Avance a la secuencia estratigráfica del 'Foso 1' de Perdiões (Reguengos de Monsaraz, Portugal) a partir de las campañas 2009 y 2010". Menga, Revista de Prehistoria de Andalucía 2: 156-175.

Márquez, J.E.; Suárez, J.; Mata Vivar, E.; jiménez, V. y Caro, J.L. (2011c): “Actividades arqueológicas de la Universidad de Málaga en el Complexo Arqueológico dos Perdigões (Reguengos de Monsaraz, Portugal). Trienio 2008-2010". Apontamentos de Arqueologia e Património 7: 33-40.
Márquez, J.E.; Valera, A.C.; Becker, H.; Jiménez, V. y Suárez, J. (2011d): “El Complexo Arqueológico dos Perdigões (Reguengos de Monsaraz, Portugal). Prospecciones Geofísicas-Campaña 2008-09”. Trabajos de Prehistoria 68(1): 175-186.

Oeschger, H.; Siegenthaler, U.; Schotterer U. y Gugelmann, A. (1975): “A box diffusion model to study the carbon dioxide exhange in nature". Tellus 27 : 168-192.

Pollard, J. (1999): "These places have their moments': thoughts on settlement practices in the British Neolithic", en J. Brück, y M. Goodman (eds.), Making places in the prehistoric world: themes in settlement archaeology, pp. 76-93. Londres, UCL Press.

Pollard, J. (2001): "The aesthetics of depositional practice". World Archaeology 33 (2): 315-333. http:// dx.doi.org/10.1080/00438240120079316

Stuiver, M. y Braziunas, T.F. (1993): "Modeling atmospheric $14 \mathrm{C}$ influences and $14 \mathrm{C}$ ages of marine samples to 10,000 BC". Radiocarbon 35(1): 137-189.

Valera, A.C. (2008a): "Intervenção arqueológica de 2007 no interior do recinto Pré-Histórico dos Perdigões (Reguengos de Monsaraz)”. Apontamentos de Arqueologia e Património 1: 15-22.

Valera, A.C. (2008b): "Recinto calcolítico dos Perdigões: fossos e fossas do Sector I". Apontamentos de Arqueologia e Património 3: 19-27.

Valera, A.C. (2009): "Construção da temporalidade nos Perdigões: contextos neolíticos na área central". Apontamentos de Arqueologia e Património 5: 17-24.

Valera, A.C.; Jorge, P. y Lago, M. (2008): “O Complexo Arqueológico dos Perdigões. Breve percurso de uma Arqueologia de minimização a uma Arqueologia em construção e em Sociedade". Almadan II/ Série 16: 115-123.

Valera, A.C. y Silva, A.M. (2011): "Dataçoes de radiocarbono para os Perdigões (1): Contextos com restos humanos nos sectores I \& Q". Apontamentos de Arqueologia e Património 7: 7-14.

Valera, A.C.; Silva, A.M. y Márquez, J.E. (en prensa): "A temporalidade dos Recintos dos Perdigões: cronologia absoluta de estruturas e práticas". VI Encuentro de Arqueología del Suroeste Peninsular, 4-6 Octubre 2012, Villafranca de los Barros, Badajoz.

Whittle, A.; Healy, F. y Bayliss, A. (2011): Gathering Time. Dating the early neolithic enclosures of Southern Britain and Ireland. Oxford, Oxbow Books. 